\title{
A strategy for building and using a human reference
}

\section{pangenome [version 1; peer review: 1 approved, 1 approved}

\section{with reservations]}

\author{
Bastien Llamas ${ }^{*}$, Giuseppe Narzisi (102*, Valerie Schneider ${ }^{3 *}$, Peter A. Audano4, \\ Evan Biederstedt ${ }^{5}$, Lon Blauvelt ${ }^{6}$, Peter Bradbury ${ }^{(107}$, Xian Chang ${ }^{6}$, \\ Chen-Shan Chin ${ }^{8}$, Arkarachai Fungtammasann ${ }^{8}$, Wayne E. Clarke ${ }^{2}$, Alan Cleary ${ }^{9}$, \\ Jana Ebler ${ }^{10}$, Jordan Eizenga ${ }^{6}$, Jonas A. Sibbesen ${ }^{6}$, Charles J. Markello6, \\ Erik Garrison6, Shilpa Garg ${ }^{11}$, Glenn Hickey ${ }^{6}$, Gerard R. Lazo12, Michael F. Lin ${ }^{13}$, \\ Medhat Mahmoud 14, Tobias Marschall10, Ilia Minkin15, Jean Monlong (iD6, \\ Rajeeva L. Musunuri2, Sagayamary Sagayaradj16,17, Adam M. Novak (D6, \\ Mikko Rautiainen 10, Allison Regier (DiD), Fritz J. Sedlazeck (D14, Jouni Siren6, \\ Yassine Souilmi (iD1, Justin Wagner ${ }^{19}$, Travis Wrightsman'20,
} Toshiyuki T. Yokoyama21, Qiandong Zeng (iD)22, Justin M. Zook ${ }^{19}$, Benedict Paten ${ }^{6}$, Ben Busby 3

\footnotetext{
${ }^{1}$ Western Regional Research Center, USDA-ARS, Albany, CA, 94710-1105, USA

${ }^{2}$ mlin.net LLC, San Jose, CA, 95113, USA

${ }^{3}$ Human Genome Sequencing Center, Department of Molecular and Human Genetics, Baylor College of Medicine, Houston TX, TX, 77030, USA

${ }^{4}$ Department of Computer Science and Engineering, The Pennsylvania State University, University Park, PA, 16802, USA

${ }^{5}$ Genome Center, University of California, Davis, Davis, CA, USA

${ }^{6}$ BASF, West Sacramento, CA, USA

${ }^{7}$ McDonnell Genome Institute, Washington University in St Louis, St Louis, MO, 63108, USA

${ }^{8}$ Material Measurement Laboratory, , National Institute of Standards and Technology, Gaithersburg, MD, 20899, USA

${ }^{9}$ Section of Plant Breeding and Genetics, Cornell University, Ithaca, NY, 14853, USA

${ }^{10}$ Department of Computational Biology and Medical Sciences, Graduate School of Frontier Sciences, The University of Tokyo, Chiba, Japan

${ }^{11}$ Laboratory Corporation of America Holdings, Westborough, MA, 01581, USA

${ }^{12}$ Australian Centre for Ancient DNA, School of Biological Sciences, Environment Institute, The University of Adelaide, Adelaide, South Australia, 5005, Australia

${ }^{13}$ New York Genome Center, New York, NY, 10013, USA

${ }^{14}$ National Center for Biotechnology Information, National Library of Medicine, National Institutes of Health, Bethesda, MD, 20894, USA

${ }^{15}$ Department of Genome Sciences, University of Washington School of Medicine, Seattle, WA, 98195, USA

${ }^{16}$ Kravis Center for Molecular Oncology, Memorial Sloan Kettering Cancer Center, New York, NY, 10065, USA

${ }^{17}$ Genomics Institute, University of California, Santa Cruz, Santa Cruz, CA, 95064, USA

${ }^{18}$ Robert W. Holley Center, USDA-ARS, Ithaca, NY, 14853, USA

${ }^{19}$ DNAnexus, Mountain View, CA, 94040, USA

${ }^{20}$ National Center for Genome Resources 87505, Santa Fe, NM, 87505, USA

${ }^{21}$ Max Planck Institute for Informatics, Saarbrücken, Germany

${ }^{22}$ Department of Genetics, Harvard Medical School, Boston, MA, 02115, USA

* Equal contributors
} 
V1 First published: 14 Oct 2019, 8:1751

https://doi.org/10.12688/f1000research.19630.1

Latest published: $29 \mathrm{Jul} 2021, \mathbf{8 : 1 7 5 1}$

https://doi.org/10.12688/f1000research.19630.2

\section{Abstract}

In March 2019, 45 scientists and software engineers from around the world converged at the University of California, Santa Cruz for the first pangenomics codeathon. The purpose of the meeting was to propose technical specifications and standards for a usable human pangenome as well as to build relevant tools for genome graph infrastructures. During the meeting, the group held several intense and productive discussions covering a diverse set of topics, including advantages of graph genomes over a linear reference representation, design of new methods that can leverage graph-based data structures, and novel visualization and annotation approaches for pangenomes. Additionally, the participants self-organized themselves into teams that worked intensely over a three-day period to build a set of pipelines and tools for specific pangenomic applications. A summary of the questions raised and the tools developed are reported in this manuscript.

Keywords

Hackathon, Pangenome, Graph Genome, RNAseq, Structural Variant

\section{Open Peer Review}

\section{Approval Status}

1

2

version 2
(revision)

1. Anna Kuosmanen (D), University of Helsinki, Helsinki, Finland

\section{Robert A. Beagrie ID, University of Oxford,}

Oxford, UK

Any reports and responses or comments on the article can be found at the end of the article.

This article is included in the Bioinformatics

gateway.

This article is included in the Max Planck Society

collection.

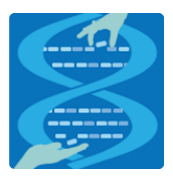

This article is included in the Hackathons

collection.

Corresponding authors: Bastien Llamas (bastien.Ilamas@adelaide.edu.au), Evan Biederstedt (evan.biederstedt@gmail.com), Benedict Paten (bpaten@ucsc.edu), Ben Busby (ben.busby@nih.gov)

Author roles: Llamas B: Formal Analysis, Investigation, Methodology, Software, Validation, Visualization, Writing - Original Draft Preparation, Writing - Review \& Editing; Narzisi G: Formal Analysis, Investigation, Methodology, Software, Validation, Visualization, Writing - Original Draft Preparation, Writing - Review \& Editing; Schneider V: Data Curation, Formal Analysis, Funding Acquisition, Investigation, Methodology, Resources, Validation, Writing - Original Draft Preparation, Writing - Review \& Editing; Audano PA: Formal Analysis, Methodology, Software, Validation, Writing - Original Draft Preparation, Writing - Review \& Editing; Biederstedt E: Formal Analysis, Methodology, Software, Validation, Writing - Original Draft Preparation, Writing - Review \& Editing; Blauvelt L: Software, 
Visualization, Writing - Original Draft Preparation, Writing - Review \& Editing; Bradbury P: Formal Analysis, Investigation, Methodology, Writing - Original Draft Preparation, Writing - Review \& Editing; Chang X: Formal Analysis, Methodology, Software, Validation, Writing Original Draft Preparation, Writing - Review \& Editing; Chin CS: Formal Analysis, Investigation, Methodology, Software, Validation, Visualization, Writing - Original Draft Preparation, Writing - Review \& Editing; Fungtammasan A: Formal Analysis, Methodology, Software, Validation, Writing - Original Draft Preparation, Writing - Review \& Editing; Clarke WE: Formal Analysis, Investigation, Methodology, Software, Validation, Visualization, Writing - Original Draft Preparation, Writing - Review \& Editing; Cleary A: Formal Analysis, Investigation, Methodology, Writing - Original Draft Preparation, Writing - Review \& Editing; Ebler J: Formal Analysis, Investigation, Methodology, Software, Validation, Visualization, Writing - Original Draft Preparation, Writing - Review \& Editing; Eizenga J: Formal Analysis, Investigation, Methodology, Software, Validation, Visualization, Writing - Original Draft Preparation, Writing - Review \& Editing; Sibbesen JA: Formal Analysis, Investigation, Methodology, Project Administration, Software, Validation, Visualization, Writing Original Draft Preparation, Writing - Review \& Editing; Markello CJ: Formal Analysis, Investigation, Methodology, Software, Validation, Visualization, Writing - Original Draft Preparation, Writing - Review \& Editing; Garrison E: Conceptualization, Formal Analysis, Investigation, Methodology, Software, Validation, Visualization, Writing - Original Draft Preparation, Writing - Review \& Editing; Garg S: Data Curation, Software, Writing - Original Draft Preparation; Hickey G: Data Curation, Methodology, Software, Validation, Visualization, Writing - Original Draft Preparation, Writing - Review \& Editing; Lazo GR: Formal Analysis, Methodology, Validation, Visualization, Writing - Original Draft Preparation, Writing - Review \& Editing; Lin MF: Data Curation, Investigation, Methodology, Software, Validation, Visualization, Writing - Original Draft Preparation, Writing - Review \& Editing; Mahmoud M: Data Curation, Investigation, Methodology, Software, Validation, Visualization, Writing - Original Draft Preparation, Writing - Review \& Editing; Marschall T: Conceptualization, Formal Analysis, Methodology, Software, Validation, Visualization, Writing - Original Draft Preparation, Writing - Review \& Editing; Minkin I: Formal Analysis, Investigation, Methodology, Software, Validation, Visualization, Writing - Original Draft Preparation, Writing Review \& Editing; Monlong J: Formal Analysis, Investigation, Methodology, Software, Validation, Visualization, Writing - Original Draft Preparation, Writing - Review \& Editing; Musunuri RL: Data Curation, Investigation, Methodology, Software, Validation, Visualization, Writing - Original Draft Preparation, Writing - Review \& Editing; Sagayaradj S: Data Curation, Investigation, Methodology, Software, Validation, Visualization, Writing - Original Draft Preparation, Writing - Review \& Editing; Novak AM: Formal Analysis, Investigation, Methodology, Software, Validation, Visualization, Writing - Original Draft Preparation, Writing - Review \& Editing; Rautiainen M: Formal Analysis, Investigation, Methodology, Software, Validation, Visualization, Writing - Original Draft Preparation, Writing - Review \& Editing; Regier A: Formal Analysis, Investigation, Methodology, Software, Validation, Visualization, Writing - Original Draft Preparation, Writing Review \& Editing; Sedlazeck FJ: Formal Analysis, Investigation, Methodology, Software, Validation, Visualization, Writing - Original Draft Preparation, Writing - Review \& Editing; Siren J: Formal Analysis, Investigation, Methodology, Software, Validation, Visualization, Writing - Original Draft Preparation, Writing - Review \& Editing; Souilmi Y: Data Curation, Investigation, Methodology, Software, Validation, Visualization, Writing - Original Draft Preparation, Writing - Review \& Editing; Wagner J: Formal Analysis, Investigation, Methodology, Software, Validation, Visualization, Writing - Original Draft Preparation, Writing - Review \& Editing; Wrightsman T: Formal Analysis, Investigation, Methodology, Software, Validation, Visualization, Writing - Original Draft Preparation, Writing - Review \& Editing; Yokoyama TT: Data Curation, Investigation, Methodology, Software, Validation, Visualization, Writing - Original Draft Preparation, Writing - Review \& Editing; Zeng Q: Formal Analysis, Investigation, Methodology, Software, Validation, Visualization, Writing - Original Draft Preparation, Writing - Review \& Editing; Zook JM: Data Curation, Formal Analysis, Investigation, Methodology, Project Administration, Software, Validation, Visualization, Writing - Original Draft Preparation, Writing - Review \& Editing; Paten B: Conceptualization, Data Curation, Methodology, Resources, Software, Supervision, Writing - Review \& Editing; Busby B: Conceptualization, Project Administration, Resources, Writing - Original Draft Preparation, Writing - Review \& Editing

Competing interests: No competing interests were disclosed.

Grant information: The work of V.A.S., B.B. and C.L. was supported by the Intramural Research Program of the National Library of Medicine, National Institutes of Health. B.L. and Y.S. were supported by the Australian Research Council. F.J.S. was supported by US National Institutes of Health (UM1 HG008898). The work of A.M.N., G.H., J.M.E., X.C., J.S., J.M., and B.P. was supported by the National Institutes of Health (5U41HG007234), the W.M. Keck Foundation (DT06172015) and the Simons Foundation (SFLIFE\# 35190). The work of J.A.S. was supported by the Carlsberg Foundation.

The funders had no role in study design, data collection and analysis, decision to publish, or preparation of the manuscript.

Copyright: $\odot 2019$ Llamas B et al. This is an open access article distributed under the terms of the Creative Commons Attribution License , which permits unrestricted use, distribution, and reproduction in any medium, provided the original work is properly cited. The author(s) is/are employees of the US Government and therefore domestic copyright protection in USA does not apply to this work. The work may be protected under the copyright laws of other jurisdictions when used in those jurisdictions.

How to cite this article: Llamas B, Narzisi G, Schneider $V$ et al. A strategy for building and using a human reference pangenome [version 1; peer review: 1 approved, 1 approved with reservations] F1000Research 2019, 8:1751

https://doi.org/10.12688/f1000research.19630.1

First published: 14 Oct 2019, 8:1751 https://doi.org/10.12688/f1000research.19630.1 


\section{Introduction}

\section{What is pangenomics?}

The current human reference genome, GRCh38 (Schneider et al., 2017), derives from a draft sequence that was constructed from a handful of individuals (Lander et al., 2001) likely of African and European ancestries (Reich et al., 2009). Today, GRCh38 captures a limited amount of additional genetic variation by providing alternative sequence representations ("alt loci") for complex or highly variable regions, such as the SMA and MAPT loci on chromosomes 5 and 17, respectively (Schneider et al., 2017), whose sequence is derived from additional DNA samples. However, analyses of other individual human genome assemblies from Europeans (Ameur et al., 2018; Audano et al., 2019; Kidd et al., 2010; Levy et al., 2007; Wheeler et al., 2008), East Asians (Audano et al., 2019; Kidd et al., 2010; Li et al., 2010; Seo et al., 2016; Shi et al., 2016), South Asians (Audano et al., 2019; Kitzman et al., 2011), Amerindians (Audano et al., 2019) and Africans (Audano et al., 2019; Kidd et al., 2010; Li et al., 2010; Sherman et al., 2019) have still revealed a substantial amount of genomic information not represented in the reference assembly. Indeed, large re-sequencing projects showed an extensive human genetic diversity, even within the genomic content captured in the reference sequence (Bycroft et al., 2018; 1000 Genomes Project Consortium et al., 2010; Mallick et al., 2016). Although GRCh38 is the most complete human reference to date, it is not clear how to construct a linear reference that can capture diversity and address population biases that impact analysis (Brandt et al., 2015; Degner et al., 2009).

Why is a pangenome representation superior to the current human reference assembly model?

The diploid structure of human DNA is not currently represented in the current reference model, which is instead an arbitrary linear combination of different haplotypes (i.e., a mosaic) from multiple individuals. A human "pangenome" is a representation of all genomic variation observed in human populations (Computational Pan-Genomics Consortium, 2018). In this context, a pangenome is a more comprehensive representation of genetic diversity than an individual diploid genome or a reference comprised of linear chromosomes built from multiple individuals, such as GRCh38. By extension, pangenomics encompasses approaches that utilize a pangenome reference. Pangenomics is designed to address the limitations of current standards, such as reference bias during the identification of genomic variants, population stratification and admixture, or ancestry-specific functional variants-among others, which impact evolutionary, agricultural and health genetics research. For example, reference bias in the sequence alignment to GRCh38 (excluding its alt loci) reduces our ability to correctly genotype regions that are likely to significantly diverge from the reference chromosome representations-e.g. immune regions such as the major histocompatibility complex (MHC) and killer cell immunoglobulin-like receptors (KIR), and the CYP2D6-8 loci involved in drug metabolism (Dilthey et al., 2015). Alignment around indels becomes more challenging as their size increases with soft-clipping being preferred over split-read alignment (Garrison et al., 2018;
Narzisi et al., 2014). Variants cannot be identified within regions completely missing from the reference sequence, many of which have been recently identified to be common across individuals (Taliun et al., 2019). Although bias and missing sequence may still persist in a pangenome, their effects should be substantially less, and may even be ameliorated by adding new content to the framework. In addition to these issues with the current reference, several studies using long reads have reported an average of 20,000 structural variants (SV) per human genome, most of which fall within repetitive elements and segmental duplications (HGSVC) (Audano et al., 2019; Chaisson et al., 2015). Many of these SVs intersect genes and regulatory elements, harbor transposable elements, and affect gene expression (Audano et al., 2019; Chiang et al., 2017). Although they are largely inaccessible to short-read sequence with current methods, these variants can be more easily re-identified using a pangenome (Chen et al., 2019; Hickey et al., 2019). Complex loci that harbor multiple repeats are also quite challenging to detect and genotype by aligning reads to a linear reference. Important disease-linked repeats, such as the CAG repeat in the HTT gene that causes HD and the CAG repeat in ATXN8 that causes Spinocerebellar ataxia type 8 (SCA8), are both flanked by other polymorphic repeats making them particularly difficult to accurately genotype. Sequence graphs offer again a general and a more flexible approach to handle these complex loci (Dolzhenko et al., 2019).

\section{What is a haplotype?}

The International HapMap Consortium defines a haplotype as "a particular combination of alleles along a chromosome" (International HapMap Consortium, 2005). A diploid individual has two haplotypes for any given genomic sequence-up to the complete genome itself-since it inherits a set of homologous chromosomes from each parent (Crawford \& Nickerson, 2005). At the population level, there may be more than two haplotypes for any given sequence. The definition of haplotype will vary in the scientific literature depending on disciplinespecific questions and applications (Hoehe, 2003). For evolutionary and population geneticists, haplotype may be short for haplotype block, which is a group of alleles that are inherited together across multiple generations and results from recombination and selection; the arrangement and length of haplotype blocks will inform about past population history (Wang et al., 2002). For medical geneticists, haplotype may represent a functional haplotype at the gene level, i.e. genetic markers linked to a disease-associated allele in so-called linkage disequilibrium, or LD (Slatkin, 2008). For livestock and crop breeders, a haplotype may be the minimal genomic region that influences a trait of interest (Hayes, et al., 2013; Qian et al., 2017). Whatever the definition of a haplotype, haplotypic information can simultaneously provide clues about population history and disease or trait association (Martin et al., 2018).

\section{Why is phasing important?}

Today's widespread use of short-read sequencing provides easy access to genotypes but does not necessarily directly inform about the parental origin of each allele. However, the real power of haplotypes resides in phasing, which is the assignment of a given combination of alleles to each homologous 
chromosome (Browning \& Browning, 2011). Beyond the methodological challenge of phasing genomes (Choi et al., 2018), the two haploid sequences in a diploid genome cannot be captured simultaneously in one linear sequence. However, a genome graph representation of a pangenome provides a spatial framework to embed multiple haplotypes at once and preserve phasing information (Paten et al., 2017). This property of graph representations of a genome is critical. At the gene scale, phasing information can be used to recognize compound heterozygosity, whereby the two homologous copies of a gene are each affected by a distinct recessive mutation (Snyder et al., 2015). Phenotype prediction depends heavily on the ability to distinguish point mutations or deletions between chromosomes (Cirulli \& Goldstein, 2010; Tewhey et al., 2011), making the retention of phasing information fundamental for the interpretation of results in a personalised medicine setting. Other applications of phasing include the inference of past population demographic history by looking at the distribution and size of haplotype blocks along chromosomes (Schiffels \& Durbin, 2014). Variant imputation also depends heavily on the availability of phasing information and becomes a key approach in large cohort studies with missing genotypes (Das et al., 2018). Finally, the sequencing of fetal cell-free DNA in maternal plasma is a very promising way to study fetal genomes in a non-invasive manner. However, it is first essential to phase haplotypes from at least one of the parents (Fan et al., 2012; Kitzman et al., 2012).

\section{Methods}

Here we describe the data sets and graph construction techniques used during the codeathon, as well as the pipelines and software that were developed.

\section{Implementation}

Graph coordinates system

To establish protocols to build pangenomic graphs from chromosome-level and ultra-long assemblies, we constructed graphs using the human reference genome GRCh38.p13, CHM1 cellline data, and two primate references: chimpanzee (PanTro PTRv2; Clint; GenBank assembly accession GCA_002880755.3) and Sumatran orangutan (PonAbe3 PABv2; Susie; GenBank assembly accession GCA_002880775.3). Additionally, we built human-only graphs using the human reference genome (GRCh38. p13; GenBank assembly accession GCA_000001405.28) and the Japanese reference genome (JG1; available at https://jmorp. megabank.tohoku.ac.jp/201902/downloads/).

We used three different methods to build the graph and explore the potential limitations and advantages of each method. The first two methods allow us to explore evolutionary questions, such as ancestral states, large structural variations between groups, and complex gene genealogies. We first created graphs based on sequences from chromosome 21 from GRCh38 (CM000683.2), Clint the chimpanzee (CM009259.2), Susie the Sumatran orangutan (CM009283.2), and CHM1 (AC244111.3, AC244144.2, AC244518.2, AC245051.3, AC245314.2, AC246819.2, AC255431.1, AC256301.1, AC277730.1, AC277802.1, AC277887.1). The first method used minimap2 (v2.16-r922) (Li, 2018) with the parameter preset asm5 to do an all-vs-all alignment of the sequences. Next, we used seqwish (6e4fe705;) to induce a graph in GFAv1 (Graphical Fragment Assembly) format, later converted to VG format (Garrison et al., 2018) for further investigation. The second method used Cactus (Paten et al., 2011), a method designed to build genome graphs of different taxa while accounting for the phylogenetic relationship between the organisms included. The generated Cactus graph in HAL format was converted to VG format using hal2vg for mapping and visualisation. In addition, we used SibeliaZ (Minkin \& Medvedev, n.d.) to build a graph from chr1 of JG1 and GRCh38.

Finally, we designed a prototype of a graph coordinates system based on previously proposed ideas (Rand et al., 2017) that streamlines the incorporation of new haplotypes into the graph, while preserving a structure that is retro-compatible with the GRCh38 linear reference coordinates (Figure 1). Such a coordinate system offers a host of advantages, as it allows easier surjection/projection of graph coordinates onto the linear reference coordinates. It also streamlines variant discovery and improves annotation portability.

\section{A faster, better short-read mapper with hit chaining}

Our work modifies vg (Garrison et al., 2018; Hickey et al., 2019) to create a fast and efficient read mapper. During the codeathon, we have improved a prototype minimizer-based mapper by adding a faster clustering function to cluster minimizer hits and hit extension logic for handling clusters that have no good full-length gapless alignment (Figure 2).

The clustering algorithm has been improved by reducing the amount of data copying in the clustering implementation. Alignments may be output from the extender if chaining is not necessary. Additionally, we have devised an improved algorithm for comparing sets of clusters.

We also implement hit chaining which allows us to deal with crossovers and indels. When the extender cannot find a fulllength gapless extension of the read alignment to some haplotype with below a threshold number of mismatches, where it previously would leave the read unaligned, it will instead now compute maximal unambiguous-path exact matches between the read and the graph's embedded haplotypes and feed them to an extension step. The extension step will trace out the haplotype segments that could connect between those matches, perform gapped alignment of the relevant read sequence against each, and take the best for each possible connection. Then the resulting multipath alignment will be linearized into an optimal gapped single-path alignment for the read.

\section{Pipeline for mapper evaluation on maize graphs}

We also sought to test a plant model, using chr 10 of maize (Zea mays) by composing a graph using vg construct and comparing it to a graph created with minimap2 and seqwish (Figure 3). Unfortunately, we were unable to perform the read mapping portion of this experiment, because we were unable 

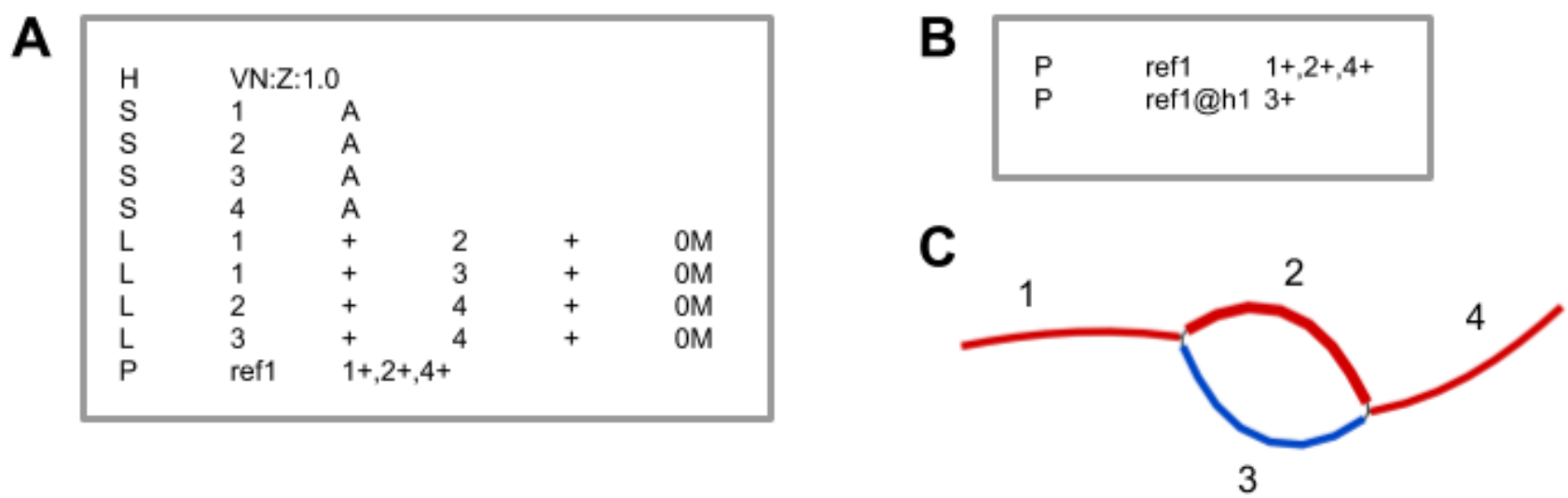

Figure 1. Proposed graph coordinate system to represent multiple haplotypes. A) Example of a GFA file (https://github.com/GFAspec/GFA-spec) that represents a reference genome and one alternate haplotype. Nodes, represented by lines starting with 'S', have a name in the second column and a nucleotide sequence in the third column. Edges, represented by lines starting with 'L', connect nodes whose sequence appears adjacent to each other in one of the haplotypes. The node names appear in the second and fourth columns, and the orientations appear in the third and fifth columns. B) A path file accompanying the GFA file includes paths for the reference genome and haplotype 1. The haplotype name is in column 2 and the sequence of nodes and their orientations are in column 3. The nucleotide sequence for any haplotype can be resolved by reading out the sequence for each node in the path. C) Visualization of $\mathbf{A}$ using path labels from $\mathbf{B}$. The red path represents ref1, while the blue path represents haplotype ref1@h1.

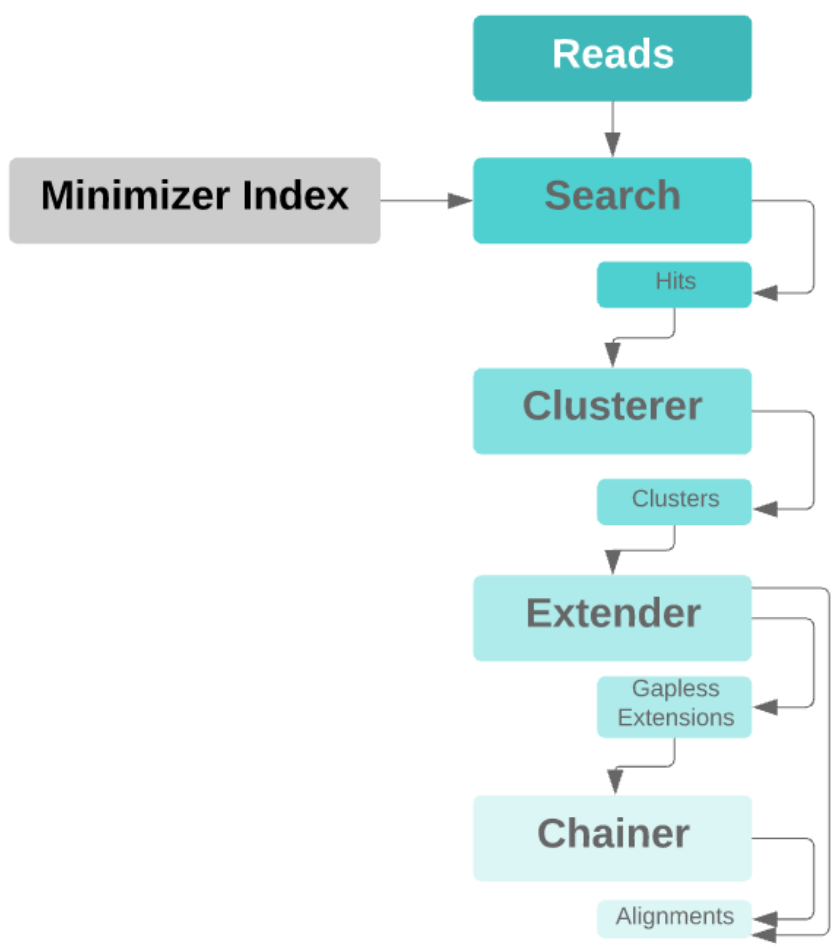

Figure 2. Pipeline diagram of the mapper. Input reads are scanned for minimizers, which are searched against a precomputed minimizer index of the graph reference. Minimizer hits for sufficiently rare minimizers are located in graph space, and the hits for all minimizers are clustered. The clusters are extended gaplessly, with a tolerance for mismatches. If a cluster produces a single full-length gapless extension, it is output as the alignment. Otherwise, partial gapless extensions are chained together by performing alignments of the intervening sequences and graph paths that connect them. 


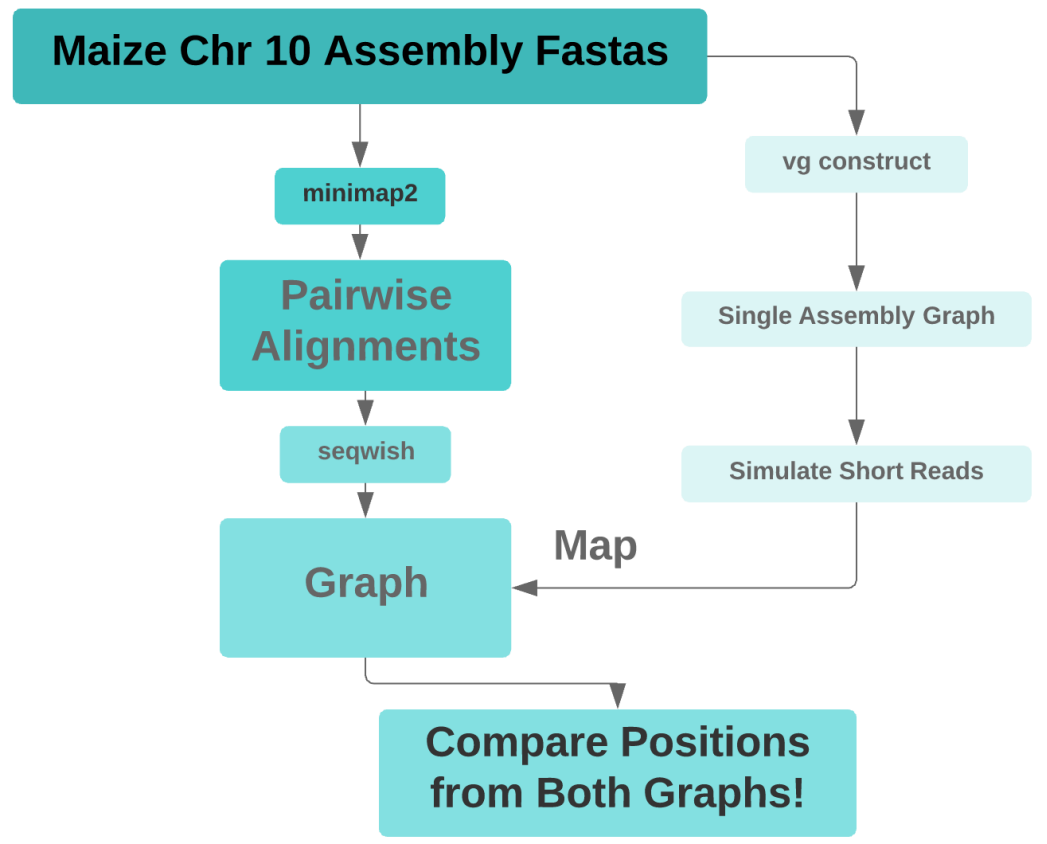

Figure 3. Pipeline diagram for mapper evaluation on Zea mays graphs. After constructing graphs with vg construct and with minimap2 and seqwish, we sought to simulate reads from the vg construct graph, align them to the minimap2/seqwish graph with our faster, better short read mapper with hit chaining, and then to evaluate the mapper's accuracy based on the simulated reads' original and realigned positions along corresponding positional paths in the two graphs.

to index the graph produced with minimap2 and seqwish for mapping. We thus aborted the experiment at that step.

We could not index the minimap2/seqwish graph for mapping because it contained extremely large snarls, with hundreds of thousands of net graph nodes. One of the indexes we needed to produce, the distance index, which is used for identifying nearby seed hits for clustering, requires doing an all-against-all distance computation on the net graph of each snarl, and that process tried to allocate more memory to hold its result than we had provisioned on our machine. We believe that the graph we generated, shown in Supplemental Figure 1 (available as Extended data) as an odgi visualization, was pathologically complex and intractable, because we did not remove spurious, short alignments from the minimap2 output. The intractability of this graph precluded further analysis.

\section{Mapping RNA sequencing data to variant graphs}

Using known variants and haplotypes during mapping of RNA sequencing (RNA-seq) data have shown to be important for reducing reference bias and thus improving downstream analyses. Reference bias is known to negatively impact estimation of allele-specific expression (Degner et al., 2009) and variant-aware mapping is one of the best ways to mitigate this problem (Castel et al., 2015). Furthermore, it has been shown that inference of gene expression in the highly polymorphic
MHC can be improved by using the alternative reference haplotypes during mapping (Lee et al., 2018). A few variant-aware methods for mapping of RNA-seq reads exist, including GNSAP (Wu \& Nacu, 2010) and Hisat2 (Kim et al., n.d.). Hisat2 is similar to $\mathrm{vg}$ in that it is also based off of a graph representation.

We wanted to test whether we could also use vg to map RNAseq reads to a graph containing both known variants, splicejunctions and haplotype-specific transcript paths. We called this a spliced variation graph. We further wanted to show that we could use the reads mapped to the graph to get unbiased estimates of allele-specific transcript expression. The pipeline would serve as a proof of concept for a graph based approach for inferring allele-specific transcript expression when an individual's haplotypes are available, similar to the personal genome approach (Rozowsky et al., 2011).

\section{Assessment of mutation rates in and around structural variants using graph genomes}

Mutation rates vary across the genome with certain hotspots associated to accessible regions as well as other genomic features. This is also discussed in the presence of the gene duplication where in a single copy gene case the mutations are rare due to the selection pressure. However, in cases in which there are two or more copies of the gene this selection 
pressure is reduced, and higher mutation rates are possible for at least one copy of the gene.

To assess the presence SNPs inside of SVs, we constructed a graph genome in vg (Garrison et al., 2018) to incorporate the SVs found in a recent Cell paper (Audano et al., 2019). This highlights one application where graph genomes might provide improved insight over traditional mapping approaches. To assess this we used SNP calls for HG002, a gold standard in genomics reported to be present based on the Genome In A Bottle (GIAB) consortium (Zook et al., 2016). We compared the power of $\mathrm{vg}$ over short Illumina reads and Pacific Biosciences (PacBio) Circular consensus sequencing (CCS) reads and PacBio continuous long reads (CLR). Subsequently we extended our project to additional samples, focusing on the assessment of mutation rates inside common SVs between the Caucasian and African populations. This revealed changes in mutation rates when looking at tandem duplications between the flanking and the affected regions. It would be interesting to scale this project further for larger cohort samples to assess the mutation rate across multiple samples and ethnicities. This would lead to a broader understanding if $\mathrm{SVs}$ are indeed the driver for certain phenotypes, or if the variations within the SVs are more likely to be impacting the phenotypes.

The code to generalize this analysis for larger cohorts such as the 1000 Genomes Project or Simons Genome Project samples is available on GitHub.

\section{Implementing annotations on pangenome graphs}

Linear genomes currently rely on genomic intervals as a core formalism for annotation but it is difficult to generalize this formalism to reference graphs. A genomic interval corresponds to a path through a graph. However, if we restrict the annotation to one path in the graph, the alternate alleles in the graph are not included in the annotation. We argue that connected subgraphs are a more appropriate formalism for annotating genome graphs. Using a new core formalism for annotation necessarily means that infrastructure to manipulate it does not yet exist. We need stable and exchangeable representations of the data, software support, and analysis tools to make the formalism useful for practitioners. We have developed a proof-of-concept system for projecting linear reference annotations onto genome graphs and utilizing them in downstream visualizers and analyses. The standard file format, named gGFF, has been defined on GitHub and code to manipulate and use this file format has been included in vg. We also developed a tool for performing utility operations on gGFF files, such as intersection and union.

A common use of annotations is generating gene or transcriptlevel counts of RNAseq read mappings for differential expression analysis. We have implemented an example RNA-seq quantification pipeline using a graph constructed from GRCh38 ch21 and variants from the 1000 Genomes Project. We converted this to a splice site-aware graph with $v g$ rna. The next step would be to map RNA-seq reads to this graph and estimate coverage per base-pair using vg pack and gene-level quantification computed using GENCODE 29 annotation.

\section{Operation}

The software should run on most Linux installations. Interested parties are encouraged to clone the GitHub repository and follow the workflow/instructions provided for the individual implementations of the Use cases listed below. Pull requests and contacting the authors is strongly encouraged.

\section{Use cases}

Graph genomes can be used for inference of extension and phasing from sparse information derived from SNP chips and RNA-seq. They can also be used to infer allele specific expression on an individual level.

There are also regions-outside the alternative loci regions that are defined for GRCh38 - that can't easily be reduced to a single reference, and assembling each individual's genome will likely be implausible for the foreseeable future.

Additionally, we are developing methods to represent variation in the clinically important MHC and to look for similarities in populations.

In theory, having clusters of haplotypes within and across populations will allow us to efficiently determine the relationships of proximal and distal phenotype-relevant events.

Taking these points together, a pangenomic graph will likely result in a reduction in the "total cost of ownership of genomes"; i.e. people can use information derived from graphs instead of remapping to a linear genome over and over again, creating novel .bams/.vcfs ad infinitum.

Use case: Integrating haplotype information into reference genome with retro-compatibility

Representing haplotype information in reference genomes is beneficial in increasing mappability and reducing bias. The major concerns for representing haplotypes in the existing reference genome are the alteration of coordinates, redundant representation, and ambiguous sequence inference. Our proposed notation tackles these issues with the following design philosophies:

1- The haplotype contigs are coordinated and defined as an add-on outside the extant reference genome coordinates. This allows the set of haplotype contigs to be updated separately, and the inclusion of haplotype sequence does not alter the underlying reference genomic coordinates. This design also allows the user to include fix patches [i.e. updates that correct errors or add sequence associated with gaps in the reference sequence; (Schneider et al., 2017)] in the graph or to recreate custom sequence using their haplotype of interest.

2- Each haplotype and nested haplotype are defined as a unique segment based on the reference genome or the 
closest haplotype; therefore, the number of bases that need to be stored for each haplotype sequence is minimalized.

3- Each haplotype can be uniquely represented using GFA-like notation that can track back into the node storing specific sequence for each haplotype.

Our proposed model allows nodes and edges represented in the GFA to change without changing the sequence corresponding to each haplotype (Figure 4).

\section{Use case: What about plants?}

Several new plant genomes have recently been sequenced and build upon the previously produced model plant assemblies, providing a foundation for research and end-use applications in agriculture. Crop plants form the foundation for the world's natural food and textile resources, and plant breeding efforts are often focused on improving several quality traits. A graph-based sequence-centric view of genomes sets the stage for facilitating key decisions that can be made to improve crop infrastructure.

Diversity in plants comprises an array of genome types with regard to species identity, genome size, chromosome number, and ploidy level. Pangenome studies have commenced for many of the model plant species, such as Arabidopsis thaliana (flowering plants) (Clark et al., 2007), Medicago truncatula (legumes) (Miller et al., 2017; Zhou et al., 2017), and Brachypodium distachyon (grasses) (Gordon et al., 2017), due to the attractive attributes of their small genomes and short generation-times. Likewise, pangenome studies have commenced on their corresponding larger cousins, which include crop plants of economic importance such as crucifers, soybean, and wheat (Montenegro et al., 2017), respectively. These previous pangenome studies used highly developed sequence analyses, but not a graph-based approach. Several pangenome-related papers appear to be in preparation for other important plant species (e.g., maize); whether they all use graph-based methods remain to be seen. The exercise of testing graph-based sequence views will help formulate use-case scenarios.

Immediate uses for graphs of plant genomes would be to validate hypothetical evolutionary tree diagrams assigned to species, and perhaps address instances where species are proposed to be ancient polyploids, or to gauge genome changes in current polyploid genomes. RNA-Seq methods may also be matched against graph-based maps to quantify expression from the genomes. For instance, it would be interesting to assess whether nutritional- or medicinal-related trait changes can be tracked to genomic structural variation using graphbased methods targeted on key metabolic pathway-associated genes. The tracking of highly repetitive transposon-initiated
A
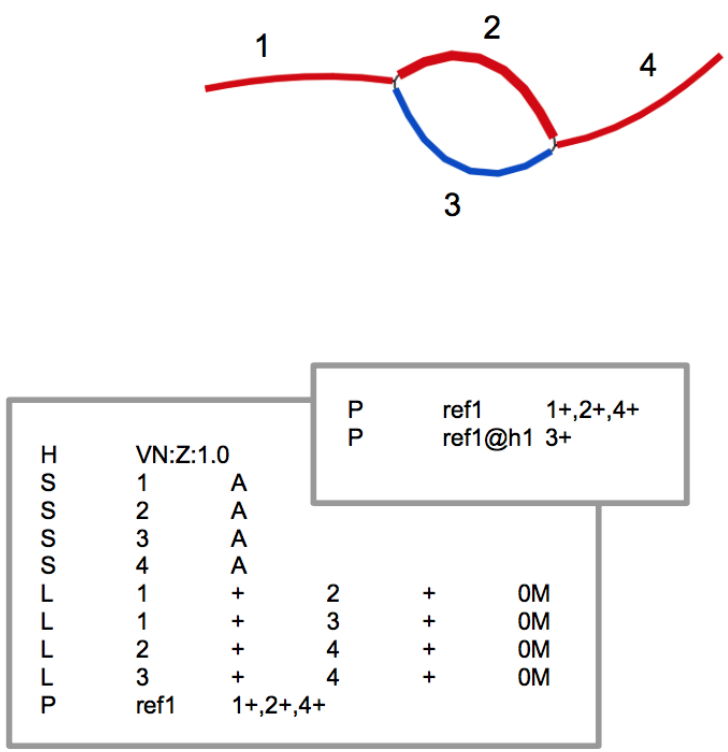

B

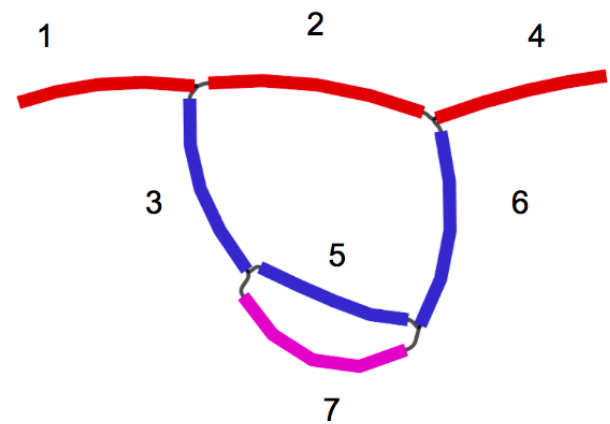

\begin{tabular}{|c|c|c|c|c|c|}
\hline $\mathrm{H}$ & \multicolumn{2}{|c|}{ VN:Z:1.0 } & $P$ & \multirow{2}{*}{\multicolumn{2}{|c|}{$\begin{array}{lr}\text { ref1 } & 1+, 2+, 4+ \\
\text { ref1@h13+,5+,6+ }\end{array}$}} \\
\hline S & 1 & A & $P$ & & \\
\hline S & 2 & A & $P$ & ref1@h1@h2 7 & $7+$ \\
\hline $\mathrm{S}$ & 3 & A & & & \\
\hline $\mathrm{S}$ & 4 & A & & & \\
\hline S & 5 & A & & & \\
\hline S & 6 & A & & & \\
\hline $\mathrm{S}$ & 7 & A & & & \\
\hline $\mathrm{L}$ & 1 & + & 2 & OM & \\
\hline $\mathrm{L}$ & 1 & + & 3 & OM & \\
\hline $\bar{L}$ & 2 & + & 4 & $\mathrm{OM}$ & \\
\hline $\mathrm{L}$ & 3 & + & 5 & OM & \\
\hline $\mathrm{L}$ & 5 & + & 6 & $\mathrm{OM}$ & \\
\hline L & 6 & + & 4 & $\mathrm{OM}$ & \\
\hline $\bar{L}$ & 3 & + & 7 & $\mathrm{OM}$ & \\
\hline $\bar{L}$ & 7 & + & 6 & $\mathrm{OM}$ & \\
\hline $\mathrm{P}$ & ref1 & & & & \\
\hline
\end{tabular}

Figure 4. Adding additional haplotype from $\mathbf{A}$ to $\mathbf{B}$. The existing sequence and coordinates remain the same even though the nodes and edges change. 
events may also explain some of the alterations observed in different genome species and their evolutionary consequence resulting in gene duplication, rearrangements, and the like. Use of graph-based methods to map out highly variable regions may also provide strategies toward implementing targeted engineering of species, or assist in classic breeding strategies where known attributes are known to structurally exist. Similarly, many wild ancestor lines are sought to bring in new gene function to serve as sources for disease resistance, quality traits, and nutrition; their inclusion in the graph will enable an understanding of their contributions on the whole genome scale. The construction of pangenomes by graph-based methods, and the subsequent visualization of these graphs therefore appear likely to have a valuable role in the future of agricultural improvements.

\section{Use case: RNA-seq mapping}

Graphs can also be used to validate and benchmark analytical methods. For example, in order to test the RNA-seq mapping performance of $\mathrm{vg}$ we created a spliced variation graph of chr21 using the rna submodule in vg (see WDL pipeline for more details). We used variants from the NA12878 individual in the 1000 Genomes Project (1000 Genomes Project Consortium et al., 2015) and transcripts from the GENCODE v29 annotation (Frankish et al., 2019). The paired-end RNA-seq reads were simulated using RSEM (Li \& Dewey, 2011) from the haplotype-specific transcripts generated from vg rna. vg's two mapping algorithms map and mpmap were able to align $71.6 \%$ and $73.8 \%$ of the simulated reads with a mapping quality of at least 30 , respectively. This is similar to the value observed for Hisat2 using the same data. We also tested both algorithms on graphs only consisting of exonic sequence. Using these graphs, the performance increased slightly (1.5 to $2 \%)$. Due to a lack of time we were not able to finish the second part of the pipeline that involved estimating allele-specific expression from the mapped reads.

This is very much a work in progress. For example, all splicejunctions and variations present in the reads were also present in the graph. In addition, due to time constraints we only used the number of mapped reads as a proxy of performance and did not assess whether the reads were correctly mapped. These issues will need to be addressed in future benchmarks in order to get a more accurate estimate of $\mathrm{vg}$ performance on spliced variation graphs.

\section{Use case: Producing a fully phased diploid assembly of the} HG002 MHC region

The MHC, located on human chr6, is a region highly enriched for genes and variation, including the human leukocyte antigen (HLA) which is involved in immune system function. Genetic associations between variants in this region involve different diseases, including autoimmune diseases. MHC haplotypes differ substantially, making it challenging to map reads from this region and call variants with conventional methods on a linear reference. We sought to generate a base-level accurate, fully phased, diploid assembly of the MHC of GIAB HG002 (NA24385, Ashkenazi son). The only previous studies producing fully phased, contiguous diploid assemblies for the MHC involved the NA12878 genome with PacBio reads (non-CCS) (Jain et al., 2018; Koren et al., 2018). In this work, we use newer PacBio CCS and ultralong Oxford Nanopore reads, along with 10x Genomics data, to produce and carefully evaluate a targeted MHC diploid assembly for a second individual from GIAB.

The data for this work relied on sequencing results from three different PacBio CCS libraries with average read lengths of $9 \mathrm{~kb}$ and $13 \mathrm{~kb}$ for the Sequel I chemistry and $11 \mathrm{~kb}$ for the Sequel II chemistry, and each dataset having $\sim 25$ to $30 \mathrm{X}$ coverage. We also used "ultralong" data from Oxford Nanopore Technology (ONT) with total coverage of 16X (4X coverage by reads $>100 \mathrm{~kb}$ ), and Promethion ONT data with total coverage of $\sim 40 \mathrm{X}$ ( $\sim 6 \mathrm{X}$ coverage by reads $>100 \mathrm{~kb})$. We also used $10 \mathrm{x}$ Genomics data for phasing. HG002 reads were extracted from the MHC (HLA1/HLA2) region on GRCh37/hg19 chr6:28,477,797-33,448,354. Illumina data for the Ashkenazi father (HG003, NA24149) and mother (HG004, NA24143) from this trio was also used to bin the CCS reads by haplotype. The HLA typing reports for HG002/HG003/HG004 were generated at Stanford Blood Center on December 16, 2016.

The first data processing step involved finding reads from each haplotype mapped to MHC regions. An initial inspection of the HG002 MHC region occurred on the whole-genome de novo assembly of trio binned reads produced using the CCS data. The MHC region initially appeared to be well-assembled, with 1 contig derived from the father and 2 contigs derived from the mother, but further inspection revealed that the results were not coherent and that some of the haplotypes may possibly having been compressed. A second approach used $15 \mathrm{~kb}$ PacBio CCS reads that were mapped to the MHC and then selecting for each haplotype. A local de novo assembly of these reads resulted in 10-15 contigs with many gaps between, although the assembly was close to the full length of the MHC. PacBio CCS reads were processed with Whatshap v0.19 to generate a phased VCF, which was then used to partition CCS reads by haplotypes. Reads for each haplotype were assembled independently into contigs that were then aligned to $10 \mathrm{x}$ Genomics Supernova assembled contigs to generate scaffolded CCS contigs for the diploid assembly. This diploid assembly was then used as the input for vg to build a genome graph via all versus all alignment (by Minimap2) followed by seqwish.

\section{Confirmation of the two haplotypes}

The CCS and ONT long reads were aligned to the genome graph to confirm the diploid assembly using the PedMEC phasing pipeline (Garg et al., 2016). In addition, phasing of HLA typing results in the diploid MHC assembly were also checked against the independent HLA typing results from Stanford Typing Lab, based on the proband phased haplotypes derived from the typing results of the parents (HG003 and HG004), as shown in Table 1 (the parents' typings are not phased).

We will continue to explore ways graph-based analyses could be used to benchmark methods used to characterize the MHC. 
Table 1. Genotyping results for proband HG002 and parents HG003 and HG004.

\begin{tabular}{|l|c|c|c|c|c|c|}
\hline \multicolumn{1}{|c|}{} & \multicolumn{2}{|c}{ Proband } & \multicolumn{2}{c|}{ Father } & \multicolumn{2}{c|}{ Mother } \\
\hline HLA & HG002 & HG002 & HG003 & HG003 & HG004 & HG004 \\
\hline A & $* 26: 01: 01: 01$ & $* 01: 01: 01: 01$ & $* 30: 01: 01$ & $* 26: 01: 01: 01$ & $* 01: 01: 01: 01$ & $* 33: 01: 01$ \\
\hline B & $* 38: 01: 01$ & $* 35: 08: 01$ & $* 13: 02: 01$ & $* 38: 01: 01$ & $* 35: 08: 01$ & $* 14: 02: 01: 01$ \\
\hline Bw & 4 & 6 & 4 & & 6 & \\
\hline Cw & $* 12: 03: 01: 01$ & $* 04: 01: 01: 06$ & $* 06: 02: 01: 01$ & $* 12: 03: 01: 01$ & $* 04: 01: 01: 06$ & $* 08: 02: 01: 01$ \\
\hline DRB1 (DR) & $* 04: 02: 01$ & $* 10: 01: 01$ & $* 07: 01: 01: 01$ & $* 04: 02: 01$ & $* 04: 04: 01$ & $* 10: 01: 01$ \\
\hline DQB1 (DQ) & $* 03: 02: 01$ & $* 05: 01: 01: 02$ & $* 02: 02: 01: 01$ & $* 03: 02: 01$ & $* 04: 02: 01$ & $* 05: 01: 01: 02$ \\
\hline DQA1 & $* 03: 01: 01$ & $* 01: 05: 01$ & $* 02: 01$ & $* 03: 01: 01$ & $* 01: 05: 01$ & $* 03: 03: 01$ \\
\hline DRB3,4,5 (DR) & $4 * 01: 03: 01: 01$ & & $4 * 01: 03: 01: 01$ & & $4 * 01: 03: 01: 01$ & \\
\hline DPA1 (DP) & $* 01: 03: 01: 04$ & $* 01: 03: 01: 02$ & $* 01: 03: 01: 04$ & $* 01: 03: 01: 05$ & $* 01: 03: 01: 02$ & $* 01: 03: 01: 04$ \\
\hline DPB1 (DP) & $* 04: 01: 01: 01$ & $* X$ & $* 04: 01: 01: 01$ & $* 04: 02: 01: 02$ & $* 04: 01: 01: 01$ & $*$ \\
\hline
\end{tabular}

It will be important to identify if these haplotypes can be represented in standard VCF files with respect to the primary GRCh37/38 references in GIAB benchmark sets, or whether existing benchmarks will need new representations and benchmarking tools. Although vg can project haplotypes into a $\mathrm{VCF}$ file with respect to the primary reference, it remains to be determined whether this is compatible with current benchmarking tools for small variants and structural variants. Other future work will entail examining whether fully phased diploid assembly is possible in other more complex, yet medically important regions, such as those of the killer-cell immunoglobulin receptor and spinal muscular atrophy.

\section{Conclusion}

Ongoing improvements in sequencing technology and diminishing costs make the generation of high-quality genome assemblies from diverse populations possible in a way today that could only have been imagined during the Human Genome Project (HGP). These new data are likely to form the basis for a new pangenome representation for the reference assembly that includes a graph, but they also raise many as-yet unanswered questions. We must consider the sample content, data/file formats that will be used, graph construction algorithms, how relevant metadata about quality and content will be communicated to users, and whether and how changes will be managed and tracked. New tools and validation sets must be built and community education will be essential, as will long-term curation, as is currently performed by the Genome Reference Consortium for the HGP reference. Ensuring the reference assembly remains a fair resource, accessible to users worldwide is also critical, and for the first time, some ethical and privacy concerns around the reference may need to be addressed. The new software developed here provide a preview of the use cases and potential for a new pangenome reference and play an important role in developing answers to these many questions.

\section{Data availability}

Underlying data

All data underlying the results are available as part of the article and no additional source data are required.

\section{Extended data}

Open Science Framework: The Human Pangenome. https://doi. org/10.17605/OSF.IO/24K9N (Busby \& Biederstedt, 2019).

Folder 'images', contained within folder 'Giraffe' contains odgi. png (Supplemental Figure 1). This file is an odgi visualization of the Zea mays chr10 minimap2/seqwish graph for two species. The pink and purple bars at the top represent regions of the linearized graph that are visited by each species' chromosome path. The black lines forming an impenetrable morass below the bars represent adjacencies between graph nodes. This graph has pathologically high connectivity.

This file is available under the MIT license.

\section{Software availability}

For graph building and observing the GRCh38 path through a primate graph, source code and directions can be found here: https://github.com/NCBI-Hackathons/ TheHumanPangenome/tree/master/DS

For ultra-fast read mapping to graph structures, source code and directions can be found here: https://github.com/ NCBI-Hackathons/TheHumanPangenome/tree/master/Giraffe

Code for converting from gff3 annotations to graph annotations can be found here: https:/github.com/ NCBI-Hackathons/TheHumanPangenome/tree/master/annotation 
WDL pipeline for mapping of RNA-seq data to spliced variant graphs can be found here:

https://github.com/NCBI-Hackathons/TheHumanPangenome/tree/ master/RNA

Code for assessing structural variants with graphs can be found here:

https://github.com/NCBI-Hackathons/TheHumanPangenome/tree/ master/SV

Code used to graph the MHC region can be found here:

https://github.com/NCBI-Hackathons/TheHumanPangenome/tree/ master/MHC
Archived source code is available at: https://doi.org/10.17605/ OSF.IO/24K9N (Busby \& Biederstedt, 2019).

\section{License: MIT License.}

Acknowledgements

Certain commercial equipment, instruments, or materials are identified to specify adequately experimental conditions or reported results. Such identification does not imply recommendation or endorsement by the National Institute of Standards and Technology, nor does it imply that the equipment, instruments, or materials identified are necessarily the best available for the purpose. We would like to thank the administrative staff of the UCSC Genome Institute, Brad Plecs, Carl Leubsdorf and the NIH STRIDES initiative.
1000 Genomes Project Consortium, Abecasis GR, Altshuler D, et al.: A map of human genome variation from population-scale sequencing. Nature. 2010; 467(7319): 1061-73.

PubMed Abstract | Publisher Full Text | Free Full Text

1000 Genomes Project Consortium, Auton A, Brooks LD, et al.: A global reference for human genetic variation. Nature. 2015; 526(7571): 68-74.

PubMed Abstract | Publisher Full Text | Free Full Text

Ameur A, Che H, Martin M, et al:: De Novo Assembly of Two Swedish Genomes Reveals Missing Segments from the Human GRCh38 Reference and Improves Variant Calling of Population-Scale Sequencing Data. Genes (Basel). 2018; 9(10): pii: E486.

PubMed Abstract | Publisher Full Text | Free Full Text

Audano PA, Sulovari A, Graves-Lindsay TA, et al.: Characterizing the Major Structural Variant Alleles of the Human Genome. Cell. 2019; 176(3): 663-75.e19. PubMed Abstract | Publisher Full Text | Free Full Text

Browning SR, Browning BL: Haplotype phasing: existing methods and new developments. Nat Rev Genet. 2011; 12(10): 703-14.

PubMed Abstract | Publisher Full Text | Free Full Text

Busby B, Biederstedt E: The Human Pangenome. 2019. http://www.doi.org/10.17605/OSF.IO/24K9N

Bycroft C, Freeman C, Petkova D, et al:: The UK Biobank resource with deep phenotyping and genomic data. Nature. 2018; 562(7726): 203-9.

PubMed Abstract | Publisher Full Tex

Castel SE, Levy-Moonshine A, Mohammadi $\mathrm{P}$, et al:: Tools and best practices for data processing in allelic expression analysis. Genome Biol. 2015; 16(1): 195. PubMed Abstract | Publisher Full Text | Free Full Text

Chaisson, MJ, Huddleston J, Dennis MY, et al.: Resolving the complexity of the human genome using single-molecule sequencing. Nature. 2015; 517(7536): 608-11.

PubMed Abstract | Publisher Full Text | Free Full Text

Chen S, Krusche P, Dolzhenko E, et al: Paragraph: A graph-based structural variant genotyper for short-read sequence data. bioRxiv. 2019. Publisher Full Text

Chiang C, Scott AJ, Davis JR, et al: The impact of structural variation on human gene expression. Nat Genet. 2017; 49(5): 692-99.

PubMed Abstract | Publisher Full Text | Free Full Text

Choi Y, Chan AP, Kirkness E, et al.: Comparison of phasing strategies for whole human genomes. PLoS Genet. 2018; 14(4): e1007308.

PubMed Abstract | Publisher Full Text | Free Full Text

Cirulli ET, Goldstein DB: Uncovering the roles of rare variants in common disease through whole-genome sequencing. Nat Rev Genet. 2010; 11(6): 415-25.

PubMed Abstract | Publisher Full Text

Clark RM, Schweikert G, Toomajian C, et al:: Common sequence polymorphisms shaping genetic diversity in Arabidopsis thaliana. Science. 2007; 317(5836) 338-42.

PubMed Abstract | Publisher Full Tex

Computational Pan-Genomics Consortium: Computational pan-genomics: status, promises and challenges. Brief Bioinform. 2018; 19(1): 118-35.

PubMed Abstract | Publisher Full Text | Free Full Text
Crawford DC, Nickerson DA: Definition and clinical importance of haplotypes. Annu Rev Med. 2005; 56: 303-20.

PubMed Abstract | Publisher Full Text

Das S, Abecasis GR, Browning BL: Genotype Imputation from Large Reference Panels. Annu Rev Genomics Hum Genet. 2018; 19: 73-96.

PubMed Abstract | Publisher Full Text

Degner JF, Marioni JC, Pai AA, et al:: Effect of read-mapping biases on detecting allele-specific expression from RNA-sequencing data. Bioinformatics. 2009; 25(24): 3207-12.

PubMed Abstract | Publisher Full Text | Free Full Text

Dilthey A, Cox C, Iqbal Z, et al:: Improved genome inference in the MHC using a population reference graph. Nat Genet. 2015; 47(6): 682-88.

PubMed Abstract | Publisher Full Text | Free Full Text

Dolzhenko E, Deshpande V, Schlesinger F, et al: ExpansionHunter: A sequencegraph based tool to analyze variation in short tandem repeat regions.

Bioinformatics. 2019; pii: btz431.

PubMed Abstract | Publisher Full Text

Fan HC, Gu W, Wang J, et al.: Non-invasive prenatal measurement of the fetal genome. Nature. 2012; 487(7407): 320-24.

PubMed Abstract | Publisher Full Text | Free Full Text

Frankish A, Diekhans M, Ferreira AM, et al:: GENCODE reference annotation for the human and mouse genomes. Nucleic Acids Res. 2019; 47(D1): D766-73. PubMed Abstract | Publisher Full Text | Free Full Text

Garg S, Martin M, Marschall T: Read-based phasing of related individuals. Bioinformatics. 2016; 32(12): i234-42.

PubMed Abstract | Publisher Full Text | Free Full Text

Garrison E, Sirén J, Novak AM, et al:: Variation graph toolkit improves read mapping by representing genetic variation in the reference. Nat Biotechnol. 2018; 36(9): 875-79.

PubMed Abstract | Publisher Full Text | Free Full Text

Gordon SP, Contreras-Moreira B, Woods DP, et al: Extensive gene conten variation in the Brachypodium distachyon pan-genome correlates with population structure. Nat Commun. 2017; 8(1): 2184.

PubMed Abstract | Publisher Full Text | Free Full Text

Hayes BJ, Lewin HA, Goddard ME: The future of livestock breeding: genomic selection for efficiency, reduced emissions intensity, and adaptation. Trends Genet. 2013; 29(4): 206-14.

PubMed Abstract | Publisher Full Text

Hickey G, Heller D, Monlong J, et al:: Genotyping Structural Variants in

Pangenome Graphs Using the vg Toolkit. bioRxiv. 2019.

Publisher Full Text

Hoehe MR: Haplotypes and the systematic analysis of genetic variation in genes and genomes. Pharmacogenomics. 2003; 4(5): 547-70.

PubMed Abstract | Publisher Full Text

International HapMap Consortium: A haplotype map of the human genome.

Nature. 2005; 437(7063): 1299-1320.

PubMed Abstract | Publisher Full Text | Free Full Text

Jain M, Koren S, Miga KH, et al.: Nanopore sequencing and assembly

of a human genome with ultra-long reads. Nat Biotechnol. 2018; 36(4) 
$338-45$

PubMed Abstract | Publisher Full Text | Free Full Text

Kidd JM, Sampas N, Antonacci F, et al.: Characterization of missing human genome sequences and copy-number polymorphic insertions. Nat Methods. 2010; 7(5): 365-71.

PubMed Abstract | Publisher Full Text | Free Full Text

Kim D, Paggi JM, Salzberg S: HISAT-Genotype: Next Generation Genomic Analysis Platform on a Personal Computer. bioRxiv. n.d. Publisher Full Text

Kitzman JO, Mackenzie AP, Adey A, et al.: Haplotype-resolved genome sequencing of a Gujarati Indian individual. Nat Biotechnol. 2011; 29(1): 59-63. PubMed Abstract | Publisher Full Text | Free Full Text

Kitzman JO, Snyder MW, Ventura M, et al.: Noninvasive whole-genome sequencing of a human fetus. Sci Transl Med. 2012; 4(137): 137ra76. PubMed Abstract | Publisher Full Text | Free Full Text

Koren S, Rhie A, Walenz BP, et al.: De novo assembly of haplotype-resolved genomes with trio binning. Nat Biotechnol. 2018. PubMed Abstract | Publisher Full Text | Free Full Text

Lander ES, Linton LM, Birren B, et al.: Initial sequencing and analysis of the human genome. Nature. 2001; 409(6822): 860-921.

PubMed Abstract | Publisher Full Text

Lee W, Plant K, Humburg P, et al:: AltHapAlignR: improved accuracy of RNAseq analyses through the use of alternative haplotypes. Bioinformatics. 2018; 34(14): 2401-8.

PubMled Abstract | Publisher Full Text | Free Full Text

Levy S, Sutton G, Ng PC, et al:: The diploid genome sequence of an individual human. PLOS Biol 2007: 5(10): e254.

PubMed Abstract | Publisher Full Text | Free Full Text

Li B, Dewey CN: RSEM: accurate transcript quantification from RNA-Seq data with or without a reference genome. BMC Bioinformatics. 2011; 12: 323. PubMed Abstract | Publisher Full Text | Free Full Text

$\mathrm{Li} \mathrm{H}$ : Minimap2: pairwise alignment for nucleotide sequences. Bioinformatics. 2018; 34(18): 3094-3100.

PubMed Abstract | Publisher Full Text | Free Full Text

$\mathrm{Li}$ R, Li Y, Zheng $\mathrm{H}$, et al.: Building the sequence map of the human pangenome. Nat Biotechnol. 2010; 28(1): 57-63.

PubMed Abstract | Publisher Full Text

Mallick S, Li H, Lipson M, et al:: The Simons Genome Diversity Project: $\mathbf{3 0 0}$ genomes from 142 diverse populations. Nature. 2016; 538(7624): 201-6. PubMed Abstract | Publisher Full Text | Free Full Text

Martin AR, Karczewski KJ, Kerminen S, et al:: Haplotype Sharing Provides Insights into Fine-Scale Population History and Disease in Finland. Am J Hum Genet. 2018; 102(5): 760-75.

PubMed Abstract | Publisher Full Text | Free Full Text

Miller JR, Zhou P, Mudge J, et al:: Hybrid assembly with long and short reads improves discovery of gene family expansions. BMC Genomics. 2017; 18(1): 541 PubMed Abstract | Publisher Full Text | Free Full Text

Minkin I, Medvedev P: Scalable Multiple Whole-Genome Alignment and Locally Collinear Block Construction with SibeliaZ bioRxiv n.d.

Publisher Full Text

Montenegro JD, Golicz AA, Bayer PE, et al:: The pangenome of hexaploid bread wheat. Plant J. 2017: 90(5): 1007-13.

PubMed Abstract | Publisher Full Text

Narzisi G, O'Rawe JA, lossifov I, et al.: Accurate de novo and transmitted indel detection in exome-capture data using microassembly. Nat Methods. 2014

11(10): 1033-36.

PubMed Abstract | Publisher Full Text | Free Full Text

Paten B, Earl D, Nguyen N, et al:: Cactus: Algorithms for genome multiple sequence alignment. Genome Res. 2011; 21(9): 1512-28.

PubMed Abstract | Publisher Full Text | Free Full Text

Paten B, Novak AM, Eizenga JM, et al.: Genome Graphs and the Evolution of
Genome Inference. Genome Res. 2017; 27(5): 665-76 PubMed Abstract | Publisher Full Text | Free Full Text

Qian L, Hickey LT, Stahl A, et al.: Exploring and Harnessing Haplotype Diversity to Improve Yield Stability in Crops. Front Plant Sci. 2017; 8: 1534.

PubMed Abstract | Publisher Full Text | Free Full Text

Rand KD, Grytten I, Nederbragt AJ, et al.: Coordinates and intervals in graphbased reference genomes. BMC Bioinformatics. 2017; 18(1): 263.

PubMed Abstract | Publisher Full Text | Free Full Text

Reich D, Nalls MA, Kao WH, et al.: Reduced neutrophil count in people of African descent is due to a regulatory variant in the Duffy antigen receptor for chemokines gene. PLoS Genet. 2009; 5(1): e1000360.

PubMed Abstract | Publisher Full Text | Free Full Text

Rozowsky J, Abyzov A, Wang J, et al:: AlleleSeq: analysis of allele-specific expression and binding in a network framework. Mol Syst Biol. 2011; 7: 522. PubMed Abstract | Publisher Full Text | Free Full Text

Schiffels S, Durbin R: Inferring human population size and separation history from multiple genome sequences. Nat Genet. 2014; 46(8): 919-25.

PubMed Abstract | Publisher Full Text | Free Full Text

Schneider VA, Graves-Lindsay T, Howe K, et al: Evaluation of GRCh38 and de novo haploid genome assemblies demonstrates the enduring quality of the reference assembly. Genome Res. 2017; 27(5): 849-64.

PubMed Abstract | Publisher Full Text | Free Full Text

Seo JS, Rhie A, Kim J, et al:: De novo assembly and phasing of a Korean human genome. Nature. 2016; 538(7624): 243-47.

PubMed Abstract | Publisher Full Text

Sherman RM, Forman J, Antonescu V, et al:: Assembly of a pan-genome from deep sequencing of 910 humans of African descent. Nat Genet. 2019; 51(1): 30-35. PubMed Abstract | Publisher Full Text | Free Full Text

Shi L, Guo Y, Dong C, et al.: Long-read sequencing and de novo assembly of a Chinese genome. Nat Commun. 2016; 7: 12065.

PubMed Abstract | Publisher Full Text | Free Full Text

Slatkin M: Linkage disequilibrium--understanding the evolutionary past and mapping the medical future. Nat Rev Genet. 2008; 9(6): 477-85.

PubMed Abstract | Publisher Full Text | Free Full Text

Snyder MW, Adey A, Kitzman JO, et al.: Haplotype-resolved genome

sequencing: experimental methods and applications. Nat Rev Genet. 2015;

16(6): 344-58.

PubMed Abstract | Publisher Full Tex

Taliun D, Harris DN, Kessler MD, et al: Sequencing of 53,831 diverse genomes from the NHLBI TOPMed Program. bioRxiv. 2019.

Publisher Full Text

Tewhey R, Bansal V, Torkamani A, et al.: The importance of phase information for human genomics. Nat Rev Genet. 2011; 12(3): 215-23.

PubMed Abstract | Publisher Full Text | Free Full Text

Wang N, Akey JM, Zhang K, et al.: Distribution of recombination crossovers and the origin of haplotype blocks: the interplay of population history,

recombination, and mutation. Am J Hum Genet. 2002; 71(5): 1227-34.

PubMed Abstract | Publisher Full Text | Free Full Text

Wheeler DA, Srinivasan M, Egholm M, et al.: The complete genome of an individual by massively parallel DNA sequencing. Nature. 2008; 452(7189): 872-76.

PubMed Abstract | Publisher Full Tex

Wu TD, Nacu S: Fast and SNP-tolerant detection of complex variants and splicing in short reads. Bioinformatics. 2010; 26(7): 873-81.

PubMed Abstract | Publisher Full Text | Free Full Text

Zhou P, Silverstein KAT, Ramaraj T, et al:: Exploring structural variation and gene family architecture with De Novo assemblies of 15 Medicago genomes. BMC Genomics. 2017; 18(1): 261.

PubMed Abstract | Publisher Full Text | Free Full Text

Zook JM, Catoe D, McDaniel J, et al: Extensive sequencing of seven human genomes to characterize benchmark reference materials. Sci Data. 2016; 160025.

PubMed Abstract | Publisher Full Text | Free Full Text 


\section{Open Peer Review}

\section{Current Peer Review Status:}

\section{Version 1}

Reviewer Report 12 November 2019

https://doi.org/10.5256/f1000research.21527.r55209

(C) 2019 Beagrie R. This is an open access peer review report distributed under the terms of the Creative Commons Attribution License, which permits unrestricted use, distribution, and reproduction in any medium, provided the original work is properly cited.

\section{Robert A. Beagrie}

MRC Molecular Haematology Unit, MRC Weatherall Institute of Molecular Medicine, Radcliffe Department of Medicine, University of Oxford, Oxford, UK

Llamas, Narzisi, Schneider et al. present the results of a pangenomics codeathon held at UCSC this March. Specifically, they detail their progress towards creating a useable human pangenome and a set of fast and reliable software tools for manipulating and working with pangenome graphs. They compare three different methods for building a pangenome graph (Minimap/Seqwish, Cactus and Sibeliaz), suggest a prototype graph coordinates system to facilitate comparison/conversion to linear reference genomes, improve short-read mapping by vg, evaluate their graph genome performance in various use cases and provide tools for annotating pangenome graphs. Useable graph genomes that incorporate known human genetic diversity would be an incredibly useful resource for a wide range of fields, so the work presented certainly should be of broad interest. Overall, the authors have made good progress on a number of fronts, especially given the limited time available during a codeathon, however I think they could do a better job of justifying their design choices, summarising their findings and outlining necessary future steps.

The manuscript starts by comparing three methods for building pangenome graphs to "explore the potential limitations and advantages of each method". I fully agree that determining the best currently available method is an important first step towards a human pangenome and progress has been made towards this goal. However, the SibeliaZ part of the pipeline built a graph using a different chromosome from the other two, which will make future comparisons much more complicated. The authors do not reach the stage where they can draw conclusions about the limitations or advantages of the different methods, but at a minimum, they should outline the future steps that would need to be taken to decide on a "best" method.

The authors propose a new graph co-ordinate system as an extension of the GFA file format, where the major difference seems to be an additional file listing the alternative haplotypes. I do not fully understand the explanation of why the new format is an improvement over GFA. The idea seems to be to allow haplotypes to be updated "separately", yet in Figure 4 both the GFA file and 
the additional haplotype file need to be altered to add a new haplotype, so what is the advantage of the separate haplotype file? Whilst the coordinates of the reference do not change from Fig $4 a$ to Fig $4 \mathrm{~b}$, the coordinates of haplotype $1 \mathrm{do}$. Would it not be important to maintain co-ordinates for all previously defined haplotypes when adding in new variants?

In summary, the selection and application of software tools and methodological approaches is scientifically sound, the questions addressed are important and interesting and the manuscript does a great job of explaining the potential benefits of a pangenome graph representation over traditional linear genomes. However, the manuscript needs some rewriting to clearly articulate what the authors have learned about best practices for constructing pangenomic graphs and what they see as the next important steps on the path to constructing a high-quality human pangenome.

Is the rationale for developing the new software tool clearly explained? Yes

Is the description of the software tool technically sound? Yes

Are sufficient details of the code, methods and analysis (if applicable) provided to allow replication of the software development and its use by others?

Yes

Is sufficient information provided to allow interpretation of the expected output datasets and any results generated using the tool?

Partly

Are the conclusions about the tool and its performance adequately supported by the findings presented in the article?

Partly

Competing Interests: No competing interests were disclosed.

Reviewer Expertise: Genomics, epigenetics, gene regulation, human genetics, sequence variation.

I confirm that I have read this submission and believe that I have an appropriate level of expertise to confirm that it is of an acceptable scientific standard, however I have significant reservations, as outlined above.

Reviewer Report 29 October 2019

https://doi.org/10.5256/f1000research.21527.r55187

(C) 2019 Kuosmanen A. This is an open access peer review report distributed under the terms of the Creative Commons Attribution License, which permits unrestricted use, distribution, and reproduction in any medium, provided the original work is properly cited. 


\section{Anna Kuosmanen}

University of Helsinki, Helsinki, Finland

The article describes the results of the first pangenomics codeathon. The purpose of the codeathon was two-fold, to propose technical specifications and standards for a usable human pangenome and to build tools for genome graphs.

The traditional representation of a reference genome is a set of linear sequences (chromosomes), with possibly additional alternative sequences to capture variations. An alternative to a linear reference genome is a "pangenome", a representation of all genomic variation observed in a population. Pangenomes are modeled as graphs in this article. The article discusses the benefits of a pangenome reference over the traditional reference, and describes several software tools and pipelines for pangenomics applications.

The authors explain very well the limitations of the linear reference genome, and describe how a pangenome graph reference would be superior. And it is great that the Conclusions section also raises important non-technical matters related to pangenomes, such as privacy concerns.

The tools and pipelines described in the article build on VG, with some of them being very much work in progress, as is natural for the results of a codeathon. All the tools and pipelines, as well as the data used in the codeathon, are described in detail, and additionally all the code is available on github, allowing for easy replication of the development. Github also has detailed instructions on the use of the tools/pipelines and examples of the output.

The article organization is at times confusing. The methods section consists of two parts: Implementation and Use cases, with the topics of Use cases and Implementation overlapping. But each category also has topics which are not in the other. The distinction between these two categories isn't clear either, as depending on the topic the data and/or methods descriptions can be found in one or the other (e.g. in "graph coordinate system", data and methods are described in Implementation, and "RNA-seq mapping" has all the data and methods in "Use cases").

In my opinion the article is scientifically sound, but it could use some re-structuring for better readability.

Minor comments:

1. For building the graphs you describe the first two methods in detail, but for third you simply say "In addition, we used SibeliaZ to build a graph...". The third method could use a sentence or two about it too.

2. Figure 1: I found this slightly confusing that there's a GFA file that has one path ("P") line, and then there's "a path file accompanying the GFA file" with two path lines (of which one is in the former).

3. In section "Pipeline for mapper evaluation on maize graphs", it is unclear what is the goal of this experiment till you look at Figure 3. The first sentence of the section also sounds odd, like there are words missing ("We also sought to test a plant model..."), and the wording of "comparing graphs" is in conflict with Figure 3 text. 
4. Typo in GSNAP ("GNSAP") in section "Mapping RNA sequencing data to variant graphs".

5. In section "Use case: Producing a fully phased diploid assembly of the HG002 MHC region", what kind of data is $10 \mathrm{X}$ Genomics data?

Is the rationale for developing the new software tool clearly explained?

Yes

Is the description of the software tool technically sound?

Yes

Are sufficient details of the code, methods and analysis (if applicable) provided to allow replication of the software development and its use by others?

Yes

Is sufficient information provided to allow interpretation of the expected output datasets and any results generated using the tool?

Yes

Are the conclusions about the tool and its performance adequately supported by the findings presented in the article?

Yes

Competing Interests: No competing interests were disclosed.

Reviewer Expertise: Bioinformatics, method development

I confirm that I have read this submission and believe that I have an appropriate level of expertise to confirm that it is of an acceptable scientific standard. 
The benefits of publishing with F1000Research:

- Your article is published within days, with no editorial bias

- You can publish traditional articles, null/negative results, case reports, data notes and more

- The peer review process is transparent and collaborative

- Your article is indexed in PubMed after passing peer review

- Dedicated customer support at every stage

For pre-submission enquiries, contact research@f1000.com 